\title{
An information-theoretic Central Limit Theorem for finitely susceptible FKG systems
}

\author{
Oliver Johnson
}

October 27, 2018

\begin{abstract}
We adapt arguments concerning entropy-theoretic convergence from the independent case to the case of FKG random variables. FKG systems are chosen since their dependence structure is controlled through covariance alone, though in the sequel we use many of the same arguments for weakly dependent random variables. As in previous work of Barron and Johnson, we consider random variables perturbed by small normals, since the FKG property gives us control of the resulting densities. We need to impose a finite susceptibility condition that is, the covariance between one random variable and the sum of all the random variables should remain finite.
\end{abstract}

\section{Introduction and notation}

Gnedenko and Korolev [ [i] discuss the relationship between probabilistic limit theorems and the increase of entropy, saying that

The formal coincidence of definitions of entropies in physics and in information theory gives rise to the question, whether analogs of the second law of thermodynamics exist in probability theory.

It is indeed striking that whilst the principle of increase of physical entropy is taken for granted, the increase of information theoretic entropy is much less 
well understood. The Gaussian is both the distribution of maximum entropy (under a variance constraint) and the limit distribution of convolutions in the Central Limit regime (which preserves variance). There is clear physical interest in asking questions such as whether entropy always increases on convolution, and whether it tends to this maximum.

By showing that the entropy tends to its maximum, we prove the Central Limit Theorem in a stronger sense than classical methods achieve. This form of convergence implies the classical weak convergence proved in Theorem 2 of Newman [13]. Lemma 5 of Takano [16] and Theorem 3.1 of Carlen and Soffer [3] also only prove weak convergence, though under different conditions. Furthermore by understanding how score functions become more linear on convolution, we gain an insight into the workings of the limit theorem, and why the Gaussian is the limiting distribution. We are able to gain some insight into the relationship between maximum entropy distributions and limit theorems in this way, and see the Central Limit Theorem in a new light.

Gnedenko and Korolev propose a programme to investigate the relationship between results like the Central Limit Theorem and maximum entropy distributions. This programme has been developed by Brown [2], Barron [1], Johnson [6] and Barron and Johnson [7], who have used information-theoretic techniques to prove the Central Limit Theorem. These papers only deal with the case of independent random variables, [2] and [1] in the case of identically distributed variables, and [6] and [7] for non-identical variables satisfying a Lindeberg-like condition.

This paper extends these results and develops new techniques to consider the case of dependent random variables satisfying the FKG inequalities. The fact that proofs of entropy-theoretic convergence have only previously existed for independent variables is unfortunate, particularly given the natural physical interest in dependent systems. In extending Barron's work, we have shown the link between physical and information-theoretic entropies holds in more generality than Gnedenko and Korolev discussed.

Definition 1.1 A set of random variables $\left\{X_{1}, X_{2}, \ldots X_{m}\right\}$ is said to be $F K G$ if $\operatorname{Cov}\left(F\left(X_{1}, X_{2}, \ldots, X_{m}\right), G\left(X_{1}, X_{2}, \ldots X_{m}\right)\right) \geq 0$ for all increasing functions $F, G$.

FKG (Fortuin-Kastelyn-Ginibre) inequalities hold for many physical models with 'positive correlation', as discussed by Newman [13. For example, in 
the Ising model with Hamiltonian $H=\sum_{j, k} J(j-k) X_{j} X_{k}-h \sum X_{j}$, the FKG inequalities hold if $J(r) \geq 0$ for all $r$. Further, FKG inequalities hold for percolation models, where $X_{j}=I$ (vertex $j$ is in an infinite cluster) and Yukawa models of Quantum Field Theory.

To obtain convergence in relative entropy we use de Bruijn's identity, which relates the relative entropy to Fisher information of perturbed random variables, which have densities we can control.

Definition 1.2 For a random variable $U$ with variance $\sigma^{2}$ and smooth density $f$, we consider the score function $\rho(u)=f^{\prime}(u) / f(u)$, the Fisher information $J(U)=\mathbb{E} \rho^{2}(U)$, and the standardised Fisher Information $J_{\mathrm{st}}(U)=$ $\sigma^{2} J(U)-1=\mathbb{E}(\sigma \rho(U)+U / \sigma)^{2} \geq 0$.

Lemma 1.3 (de Bruijn) For $U$ with mean 0, variance 1 and density $f$, the relative entropy distance $D(f \| \phi)$ from a standard normal can be expressed in terms of the Fisher information of $U$ perturbed by normals $Z^{(\tau)} \sim N(0, \tau)$ :

$$
D(f \| \phi)=\frac{1}{2} \int_{0}^{\infty}\left(J\left(U+Z^{(\tau)}\right)-\frac{1}{1+\tau}\right) d \tau=\frac{1}{2} \int_{0}^{\infty} \frac{J_{\mathrm{st}}\left(U+Z^{(\tau)}\right)}{1+\tau} d \tau .
$$

Lemma 3 of Newman 13 shows that for $(S, T)$ FKG, we can control $\operatorname{Cov}(f(S), g(T))$. In our case this is useful because for $Z_{S}^{(\tau)}, Z_{T}^{(\tau)}$ are normal $N(0, \tau)$, independent of $S, T$ and each other, this means we can control the densities $p_{X, Y}-p_{X} p_{Y}$, where $(X, Y)=\left(S+Z_{S}^{(\tau)}, T+Z_{T}^{(\tau)}\right)$. See Lemma 3.5 for a discussion of these methods.

First we establish conditions under which convergence $J_{\text {st }}(U) \rightarrow 0$ holds, which implies more conventional forms of convergence:

Lemma 1.4 (Shimizu [14]) If $U$ has variance $\sigma^{2}$, density $f$ and distribution function $F$ then denoting the density and distribution function of a $N\left(0, \sigma^{2}\right)$ by $\phi$ and $\Phi$ respectively:

$$
\begin{aligned}
\sup _{u}|F(u)-\Phi(u)| \leq \int|f(u)-\phi(u)| d u & \leq 4 \sqrt{3} \sqrt{J_{\mathrm{st}}(U)} \\
\sup _{u}|f(u)-\phi(u)| & \leq\left(1+\sqrt{\frac{6}{\pi}}\right) \sqrt{J_{\mathrm{st}}(U)}
\end{aligned}
$$


Indeed weak convergence implies that $\lim _{n} \mathbb{E} h\left(S_{n}\right)=\mathbb{E} h(Z)$, for all bounded uniformly continuous functions $h$. Convergence in relative entropy extends this to the class of measurable functions bounded by some multiple of $x^{2}+1$ (see Barron [1] for further details).

Definition 1.5 Consider a stationary d-dimensional system of random variables $X_{\mathbf{u}}$ (where the index $\mathbf{u} \in \mathbb{Z}^{d}$ ), with mean zero and finite variance. For a particular vector $\mathbf{x}=\left(u_{1}, u_{2}, \ldots, u_{d}\right)$, we define the box

$$
B_{\mathbf{u}}=\left\{\mathbf{y}: 0 \leq y_{i} \leq u_{i} \text { for all } i\right\} .
$$

with volume $|\mathbf{u}|=\left|B_{\mathbf{u}}\right|=\prod_{i} u_{i}$. We can define $v(\mathbf{x})=\operatorname{Var}\left(\sum_{\mathbf{u} \in B_{\mathbf{x}}} X_{\mathbf{u}}\right)$ and $U_{\mathbf{x}}=\left(\sum_{\mathbf{u} \in B_{\mathbf{x}}} X_{\mathbf{u}}\right) / \sqrt{|\mathbf{x}|}$. Define perturbed random variables $Y_{\mathbf{u}}^{(\tau)}=$ $X_{\mathbf{u}}+Z_{\mathbf{u}}^{(\tau)}$, for $Z_{\mathbf{u}}^{(\tau)}$ a sequence of $N(0, \tau)$ independent of $X_{\mathbf{u}}$ and each other. We introduce $V_{\mathbf{x}}^{(\tau)}=\left(\sum_{\mathbf{u} \in B_{\mathbf{x}}} Y_{\mathbf{u}}^{(\tau)}\right) / \sqrt{|\mathbf{x}|} \sim U_{\mathbf{x}}+Z^{(\tau)}$.

\section{Condition 1 (Finite Susceptibility)}

$$
v=\sum_{\mathbf{u}} \operatorname{Cov}\left(X_{\mathbf{0}}, X_{\mathbf{u}}\right)<\infty
$$

Definition 1.6 For function $\psi$, define the class of random variables $X$ with variance $\sigma^{2}$ such that:

$$
\mathcal{C}_{\psi}=\left\{X: \mathbb{E} X^{2} I(|X| \geq R \sigma) \leq \sigma^{2} \psi(R) \text { for all } R\right\} .
$$

Condition 2 (Uniform Integrability) There exists $\psi$ such that $V_{\mathbf{u}}^{(\tau)} \in \mathcal{C}_{\psi}$ for all $\mathbf{u}$.

Theorem 1.7 Consider a stationary collection of mean zero, finite variance random variables $X_{\mathbf{u}}$ obeying the FKG inequalities and finite susceptibility (Condition [1). Then

$$
\lim _{n \rightarrow \infty}\left(\sup _{\mathbf{x}:|\mathbf{x}|=n} J_{\mathrm{st}}\left(V_{\mathbf{x}}^{(\tau)}\right)\right)=0,
$$

if and only if Condition $\mathrm{G}$ (Uniform Integrability) holds. 
Condition 2 (for stationary FKG variables with finite variance) is actually implied by Condition 1. This follows by Newman's proof 13 that these conditions imply the Central Limit Theorem, since if $F_{n}(x)$ is the distribution function of $V_{n}^{(\tau)}$, then $F_{n}(x) \rightarrow \Phi(x)$, so $\int z^{2} I(|z|>N) d F_{n}(z)=1-$ $\int z^{2} d F_{n}(z) I(|z| \leq N) d z \rightarrow 1-\int z^{2} I(|z| \leq N) d \Phi(z)=\int z^{2} I(|z|>N) d \Phi(z)$. Carlen and Soffer claim on Page 369 that if $\operatorname{Cov}\left(X_{\mathbf{0}}, X_{\mathbf{i}}\right)$ decays at a rate of $|\mathbf{i}|^{-t}$, where $t>2 d, d$ the dimension of the lattice, then Condition 2 will hold. This roughly corresponds to requiring that $\sum_{\mathbf{i}} \operatorname{Cov}\left(X_{\mathbf{0}}, X_{\mathbf{i}}\right)^{1 / 2}<\infty$.

Note: we do not need to assume that the $X_{i}$ themselves have densities - even if not, by the following Lemma we obtain weak convergence of the normalized sums of the original variables.

Definition 1.8 Define $\kappa(n, \tau)=\sup _{|\mathbf{u}| \geq n} J_{\mathrm{st}}\left(V_{\mathbf{u}}^{(\tau)}\right)$.

Condition 3 For some $n, \int \kappa(n, \tau) /(1+\tau) d \tau$ is finite.

Theorem 1.9 Consider a stationary collection of mean zero, finite variance random variables $X_{\mathbf{u}}$ with densities, obeying the FKG inequalities and Conditions 1 and 國. Then if $g_{\mathbf{u}}$ is the density of $V_{\mathbf{u}}^{(\tau)}$, then:

$$
D\left(g_{\mathbf{u}} \| \phi\right) \rightarrow 0,
$$

if and only if Condition $\mathrm{g}$ (Uniform Integrability) holds.

Proof Via monotone convergence: $\kappa(n, \tau)$ converges monotonically to 0 in $n$, and hence $\int \kappa(n, \tau) d \tau$ converges to zero.

Newman claims that if instead of scaling by $|\mathbf{x}|$, we scale by $v(\mathbf{x})$, Condition 11 can be relaxed to Condition 2 and Condition 1 .

Condition 4 If $K(R)=\sum_{|j| \leq R} \operatorname{Cov}\left(X_{0}, X_{j}\right)$, then $K(R)$ is slowly varying (that is, for any $\lambda, \lim _{R \rightarrow \infty} K(\lambda R) / K(R)=1$ ).

He remarks that Condition 2 can be checked if for example $\mathbb{E}\left(V_{n}^{(\tau)}\right)^{4} \leq$ $3\left(\mathbb{E}\left(V_{n}^{(\tau)}\right)^{2}\right)^{2}$, which itself holds in many cases as a consequence of results such as the Lebowitz inequality [9] or the GHS inequality [12]. 
Takano [15], [16] deals with the behaviour of entropy, under a $\delta_{4}$-mixing condition which seems hard to check in most useful cases, since it is defined by a ratio of densities. Further, Takano only proves convergence of in relative entropy of the 'rooms' (in Bernstein's terminology), equivalent to weak convergence of the original variables. Our conclusion holds in the stronger sense of relative entropy convergence of the full sequence. Another paper to use entropy-theoretic methods in the dependent case is by Carlen and Soffer [3]. They use a variety of conditions which are different to ours, but again only prove weak convergence for dependent variables.

\section{Fisher Information and convolution}

In the independent case, Fisher information is a sub-additive quantity on convolution. In the dependent case, we prove that Fisher information is 'almost sub-additive' - the interest comes in trying to bound the error term. Takano [15], [16] produces bounds which depend on his $\delta_{4}$ mixing coefficient, which is hard to understand, and hard to check since it depends on ratios of densities. Our calculations provide weaker, and more standard conditions under which the CLT will hold in the sense of convergence of Fisher Information.

Definition 2.1 For random variables $X, Y$ with score functions $\rho_{X}, \rho_{Y}$, for any $\beta$, we define $\widetilde{\rho}$ for the score function of $\sqrt{\beta} X+\sqrt{1-\beta} Y$ and then:

$\Delta(X, Y, \beta)=\mathbb{E}\left(\sqrt{\beta} \rho_{X}(X)+\sqrt{1-\beta} \rho_{Y}(Y)-\widetilde{\rho}(\sqrt{\beta} X+\sqrt{1-\beta} Y)\right)^{2} \geq 0$.

The principal theorem of this section is:

Theorem 2.2 Let $S$ and $T$ be FKG random variables, with mean zero and variance $\leq K$. Consider $Z_{S}^{(\tau)}$ and $Z_{T}^{(\tau)}$, distributed as $N(0, \tau)$, indepdendent of $S, T$ and of each other. Define $X=S+Z_{S}^{(\tau)}$ and $Y=T+Z_{T}^{(\tau)}$, with score functions $\rho_{X}$ and $\rho_{Y}$. There exists a constant $C=C(K, \tau, \epsilon)$ such that for any $\beta$ :

$\beta J(X)+(1-\beta) J(Y)-J(\sqrt{\beta} X+\sqrt{1-\beta} Y)+C \operatorname{Cov}(S, T)^{1 / 3-\epsilon} \geq \Delta(X, Y, \beta)$.

If $S, T$ have bounded $(2+\delta)$ th moment, we can replace $1 / 3$ by $(2+\delta) /(6+\delta)$. 
Proof The proof of the first result requires some involved analysis, and is deferred to Section 3 .

Next, we need lower bounds on the term $\Delta(X, Y, \beta)$. As discussed in Barron and Johnson [7], in the case of independent variables, such terms are equal to zero exactly when all the functions concerned are linear. In general, if such an expression is small, then the derivatives of $\rho_{X}$ and $\rho_{Y}$ are close to constant, so long as we have uniform control over the tails of $X$ and $Y$.

Proposition 2.3 For any $\psi$, there exists a function $\nu=\nu_{\psi}$, with $\nu(\epsilon) \rightarrow 0$ as $\epsilon \rightarrow 0$, such that if $X, Y$ lie in $\mathcal{C}_{\psi}$, then

$$
\beta(1-\beta) J_{\mathrm{st}}(X) \leq \nu(\Delta(X, Y, \beta)) .
$$

Proof Define a semi-norm \|\|$_{\Theta}$ on functions via:

$$
\|f\|_{\Theta}^{2}=\inf _{a, b} \mathbb{E}\left(f\left(Z_{\tau / 2}\right)-a Z_{\tau / 2}-b\right)^{2},
$$

where $Z_{\tau / 2}$ is $N(0, \tau / 2)$.

Using Lemma 3.1 of Johnson [8], for $K>0$, there exists a constant $\xi_{K}>0$ such that for any dependent random variables $(S, T)$ with variances $\leq K$ then the sum $(X, Y)=\left(S+Z_{S}^{(\tau)}, T+Z_{T}^{(\tau)}\right)$ has joint density $p^{(\tau)}(x, y)$ bounded below by $\xi_{K} \phi_{\tau / 2}(x) \phi_{\tau / 2}(y)$.

Hence writing $h(x, y)=\sqrt{\beta} \rho_{X}(x)+\sqrt{1-\beta} \rho_{Y}(y)-\widetilde{\rho}(\sqrt{\beta} x+\sqrt{1-\beta} y)$, then:

$$
\begin{aligned}
\Delta(X, Y, \beta) & =\int p^{(\tau)}(x, y) h(x, y)^{2} d x d y \geq \xi_{K} \int \phi_{\tau / 2}(x) \phi_{\tau / 2}(y) h(x, y)^{2} d x d y \\
& \geq \frac{\beta(1-\beta) \xi_{K}}{2}\left(\left\|\rho_{X}\right\|_{\Theta}^{2}+\left\|\rho_{Y}\right\|_{\Theta}^{2}\right),
\end{aligned}
$$

by Proposition 3.2 of [6]. The crucial result of Johnson [6] implies for a fixed $\psi$, if the sequence $X_{n} \in \mathcal{C}_{\psi}$ have score functions $f_{n}$, then $\left\|f_{n}\right\|_{\Theta} \rightarrow 0$ implies that $J_{\mathrm{st}}\left(X_{n}\right) \rightarrow 0$.

Define

$$
J(n)=\sup \left\{J_{\text {st }}\left(V_{\mathbf{x}}^{(\tau)}\right):|\mathbf{x}|=n\right\} .
$$

Note that in the 1-dimensional case, there is only one set of this form, $\{1,2, \ldots n\}$. 
Corollary 2.4 If Condition 6 holds for some $\psi$ then there exists $d(m) \rightarrow 0$ as $m \rightarrow \infty$ such that for $m \geq n$ :

$$
J(n+m) \leq \frac{m}{m+n} J(m)+\frac{n}{m+n} J(n)+d(m)-\nu_{\psi}^{-1}\left(\frac{J(m) m n}{(m+n)^{2}}\right) .
$$

Proof For any $\mathbf{x}$, we can decompose the box into smaller distinct ones: $B_{\mathbf{x}}=B_{\mathbf{y}} \cup \widetilde{B}_{\mathbf{z}}$, where $B_{\mathbf{y}} \cap \widetilde{B}_{\mathbf{z}}=\emptyset$, and $\mathbf{x}=\mathbf{y}=\mathbf{z}$ for all but the $j$ th coordinate, so that:

$$
B_{\mathbf{z}}=\left\{\mathbf{u}: 0 \leq u_{i} \leq x_{i} \text { for all } i \neq j \text { and } y_{j}+1 \leq u_{j} \leq y_{j}+z_{j}=x_{j}\right\} .
$$

This corresponds to splitting the box into two smaller ones by making a cut parallel to the $j$ th face. We write $\widetilde{U}_{\mathbf{z}}=\left(\sum_{\mathbf{u} \in \widetilde{B}_{\mathbf{z}}} X_{\mathbf{u}}\right) / \sqrt{|\mathbf{z}|}$, and $\widetilde{V}_{\mathbf{z}}^{(\tau)}=$ $\left(\sum_{\mathbf{u} \in \widetilde{B}_{\mathbf{z}}} Y_{\mathbf{u}}^{(\tau)}\right) / \sqrt{|\mathbf{z}|}$

Taking $\beta=m /(m+n)=|\mathbf{y}| /|\mathbf{x}|$, and by substituting in Theorem 2.2, since $J(m) \leq 1 / \tau$, we obtain

$$
\begin{aligned}
J_{\mathrm{st}}\left(V_{\mathbf{x}}^{(\tau)}\right) \leq & \frac{m}{m+n} J_{\mathrm{st}}\left(V_{\mathbf{y}}^{(\tau)}\right)+\frac{n}{n+m} J_{\mathrm{st}}\left(V_{\mathbf{z}}^{(\tau)}\right) \\
& +C^{\prime} \operatorname{Cov}\left(V_{\mathbf{y}}^{(\tau)}, \widetilde{V}_{\mathbf{z}}^{(\tau)}\right)^{1 / 3-\epsilon}-\Delta\left(V_{\mathbf{y}}^{(\tau)}, \widetilde{V}_{\mathbf{z}}^{(\tau)}, \frac{m}{m+n}\right) .
\end{aligned}
$$

Define

$$
c(m, n)=\sup \left\{\operatorname{Cov}\left(U_{\mathbf{y}}, \widetilde{U}_{\mathbf{z}}\right):|\mathbf{y}|=m,|\mathbf{z}|=n\right\} .
$$

Under the finite susceptibility condition (Condition 1), Lemma 4 of Newman 13 shows that this quantity is bounded above in a suitable way, since $\operatorname{Var}\left(U_{\mathbf{u}}-U_{\mathbf{v}}\right) \rightarrow 0$ if $|\mathbf{u}| /|\mathbf{v}| \rightarrow 0$.

We are able to complete the proof of the CLT, under FKG conditions.

Proof of Theorem 1.7 We first establish convergence along the 'powers of 2 subsequence'. Condition 2 implies that $V_{n}^{(\tau)} \in \mathcal{C}_{\psi}$ for some $\psi$ and hence that $J\left(V_{n}^{(\tau)}\right) \leq \nu_{\psi}\left(\Delta\left(V_{n}^{(\tau)}, \widetilde{V}_{n}^{(\tau)}, 1 / 2\right)\right)$. We can write $D(k)=\nu^{-1}\left(J_{\mathrm{st}}\left(V_{2^{k}}^{(\tau)} / 4\right)\right)$. By Corollary 2.4, we know that:

$$
J\left(2^{k+1}\right) \leq J\left(2^{k}\right)+d(k)-D(k),
$$

where $d(k) \rightarrow 0$.

We use an argument structured like Linnik's proof [11]. Given $\epsilon$, we can find $K$ such that $d(k) \leq \epsilon / 2$, for all $k \geq K$. Now either: 
1. For all $k \geq K, 2 d(k) \leq D(k)$, so $J\left(2^{k}\right)-J\left(2^{k+1}\right) \geq D(k) / 2$, and summing the telescoping sum, we deduce that $\sum_{k} D(k)$ is finite, and hence there exists $L$ such that $D(L) \leq \epsilon$.

2. Otherwise for some $L \geq K, 2 d(L) \geq D(L)$, then $D(L) \leq \epsilon$.

Thus, in either case, there exists $L$ such that $D(L) \leq \epsilon$, and hence by Proposition 2.3, $J\left(2^{L}\right) \leq 4 \nu(\epsilon)$.

Now, for any $k \geq L$, either $J\left(2^{k+1}\right) \leq J\left(2^{k}\right)$, or $D(k) \leq d(k) \leq \epsilon$. In the second case, $J\left(2^{k}\right) \leq 4 \nu(\epsilon)$, so that $J\left(2^{k+1}\right) \leq 4 \nu(\epsilon)+\epsilon$. In either case, we prove by induction that for all $k \geq L$, that $J\left(2^{k+1}\right) \leq 4 \nu(\epsilon)+\epsilon$.

Now, we can 'fill in the gaps' to gain control of the whole sequence, adapting the proof of the standard sub-additive inequality, using the methods described in Appendix 2 of [5].

\section{Proof of sub-additive relation}

This is the key part of the argument, proving the bounds at the heart of the limit theorems. However, although the analysis is somewhat involved, it is not too technically difficult.

We introduce notation where it will be clear whether densities or score functions are associated with joint or marginal distributions, by their number of arguments: $\rho_{X}(x)$ will be the score function of $X$, and $p_{X}^{\prime}(x)$ the derivative of its density. For joint densities $p_{X, Y}(x, y), p_{X, Y}^{(1)}(x, y)$ will be the derivative of the density with respect to the first argument and $\rho_{X, Y}^{(1)}(x, y)=$ $p_{X, Y}^{(1)}(x, y) / p_{X, Y}(x, y)$, and so on.

Note that a similar equation to the independent case tells us about the behaviour of Fisher Information of sums:

Lemma 3.1 If $X, Y$ are random variables, with joint density $p(x, y)$, and score functions $\rho_{X, Y}^{(1)}$ and $\rho_{X, Y}^{(2)}$ then $X+Y$ has score function $\widetilde{\rho}$ given by

$$
\widetilde{\rho}(z)=\mathbb{E}\left[\rho_{X, Y}^{(1)}(X, Y) \mid X+Y=z\right]=\mathbb{E}\left[\rho_{X, Y}^{(2)}(X, Y) \mid X+Y=z\right] .
$$


Proof Since $X+Y$ has density $p_{X+Y}$ given by $p_{X+Y}(z)=\int p(z-y, y) d y$, then:

$$
p_{X+Y}^{\prime}(z)=\int \frac{\partial p}{\partial z}(z-y, y) d y .
$$

Hence dividing, we obtain that:

$$
\widetilde{\rho}(z)=\frac{p_{X+Y}^{\prime}(z)}{p_{X+Y}(z)}=\int \rho_{X, Y}^{(1)}(z-y, y) \frac{p(z-y, y)}{p_{X+Y}(z)} d y,
$$

as claimed.

For given $a, b$, define the function $M(x, y)=M_{a, b}(x, y)$ by:

$$
M(x, y)=a\left(\rho_{X, Y}^{(1)}(x, y)-\rho_{X}(x)\right)+b\left(\rho_{X, Y}^{(2)}(x, y)-\rho_{Y}(y)\right),
$$

which is zero if $X$ and $Y$ are independent. We will show that if $\operatorname{Cov}(X, Y)$ is small, then $M$ is close to zero.

Proposition 3.2 If $X, Y$ are random variables, with score functions $\rho_{X}, \rho_{Y}$, and if the sum $\sqrt{\beta} X+\sqrt{1-\beta} Y$ has score function $\widetilde{\rho}$ then

$$
\begin{aligned}
\beta J(X) & +(1-\beta) J(Y)-J(\sqrt{\beta} X+\sqrt{1-\beta} Y) \\
& +2 \sqrt{\beta(1-\beta)} \mathbb{E} \rho_{X}(X) \rho_{Y}(Y)+\mathbb{E} M_{\sqrt{\beta}, \sqrt{1-\beta}}(X, Y) \widetilde{\rho}(X+Y) \\
= & \mathbb{E}\left(\sqrt{\beta} \rho_{X}(X)+\sqrt{1-\beta} \rho_{Y}(Y)-\widetilde{\rho}(\sqrt{\beta} X+\sqrt{1-\beta} Y)\right)^{2}
\end{aligned}
$$

Proof By the two-dimensional version of Stein's equation, for any function $f(x, y)$ :

$$
\mathbb{E} \rho_{X, Y}^{(1)}(X, Y) f(X, Y)=-\mathbb{E} \frac{\partial f}{\partial x}(X, Y) .
$$

In particular, if $f(x, y)=\widetilde{\rho}(x+y)$ :

$$
\mathbb{E} \rho_{X}(X) \widetilde{\rho}(X+Y)=-\mathbb{E} \widetilde{\rho}(X+Y)-\mathbb{E}\left(\rho_{X, Y}^{(1)}(X, Y)-\rho_{X}(X)\right) \widetilde{\rho}(X+Y) .
$$

Hence, we know that for any $a, b$ :

$\mathbb{E}\left(a \rho_{X}(X)+b \rho_{Y}(Y)\right) \widetilde{\rho}(X+Y)=(a+b) J(X+Y)-\mathbb{E} M_{a, b}(X, Y) \widetilde{\rho}(X+Y)$. 
By considering $\int p(x, y)\left(a \rho_{X}(x)+b \rho_{Y}(y)-(a+b) \widetilde{\rho}(x+y)\right)^{2} d x d y$, dealing with the cross term with the expression above, we deduce that:

$$
\begin{aligned}
a^{2} J(X)+b^{2} J(Y)-(a+b)^{2} J(X+Y) & \\
& +2 a b \mathbb{E} \rho_{X}(X) \rho_{Y}(Y)+2(a+b) \mathbb{E} M_{a, b}(X, Y) \widetilde{\rho}(X+Y) \\
= & \mathbb{E}\left(a \rho_{X}(X)+b \rho_{Y}(Y)-(a+b) \widetilde{\rho}(X+Y)\right)^{2} \geq 0 .
\end{aligned}
$$

As in the independent case, we can rescale, and consider $X^{\prime}=\sqrt{\beta} X, Y^{\prime}=$ $\sqrt{1-\beta} Y$, and take $a=\beta, b=1-\beta$. Note that $\sqrt{\beta} \rho_{X^{\prime}}(u)=\rho_{X}(u / \sqrt{\beta})$, $\sqrt{1-\beta} \rho_{Y^{\prime}}(v)=\rho_{Y}(v / \sqrt{1-\beta})$.

We will show that the two terms on the second line of Proposition 3.2 can be controlled when $(X, Y)=\left(S+Z_{S}^{(\tau)}, T+Z_{T}^{(\tau)}\right)$, by controlling $\operatorname{Cov}(S, T)$ alone. We need control of the score functions of perturbed variables. We obtain this in two regions, firstly in Lemma 3.3 over the tail, and then in Lemma 3.4 over the rest of the real line.

We require an extension of Lemma 3 of Barron [1] applied to single and bivariate random variables:

Lemma 3.3 For any random variables $S, T$ as before we define $(X, Y)=$ $\left(S+Z_{S}^{(\tau)}, T+Z_{T}^{(\tau)}\right)$ and define $p_{U, V}^{(2 \tau)}$ for the density of $(U, V)=\left(S+Z_{S}^{(2 \tau)}, T+\right.$ $\left.Z_{T}^{(2 \tau)}\right)$. Now there exists a constant $c_{\tau, k}=\sqrt{2}(2 k / \tau e)^{k / 2}$ such that for all $x$ :

$$
\begin{aligned}
p_{X}^{(\tau)}(x)\left|\rho_{X}(x)\right|^{k} & \leq c_{\tau, k} p_{U}^{(2 \tau)}(x) \\
p^{(\tau)}(x, y)\left|\rho_{X, Y}^{(1)}(x, y)\right|^{k} & \leq c_{\tau, k} p_{U, V}^{(2 \tau)}(x, y) \\
p^{(\tau)}(x, y)\left|\rho_{X, Y}^{(2)}(x, y)\right|^{k} & \leq c_{\tau, k} p_{U, V}^{(2 \tau)}(x, y)
\end{aligned}
$$

and hence

$$
\left(\mathbb{E}\left|\rho_{X}(X)\right|^{k}\right)^{1 / k} \leq \sqrt{\frac{2^{1 / k} 2 k}{\tau e}} .
$$

Proof We adapt Barron's proof, using Hölder's inequality and the bound; $(u / \tau)^{k} \phi_{\tau}(u) \leq c_{\tau, k} \phi_{2 \tau}(u)$ for all $u$.

$$
\begin{aligned}
p_{X}^{\prime}(x)^{k} & =\left(\mathbb{E}\left(\frac{x-S}{\tau}\right) \phi_{\tau}(x-S)\right)^{k} \\
& \leq\left(\mathbb{E}\left(\frac{x-S}{\tau}\right)^{k} \phi_{\tau}(x-S)\right)\left(\mathbb{E} \phi_{\tau}(x-S)\right)^{k-1} \\
& \leq c_{\tau, k}\left(\mathbb{E} \phi_{2 \tau}(x-S)\right) p_{X}(x)^{k-1}
\end{aligned}
$$


A similar argument gives the other bounds.

Now, the normal perturbation ensures that the density does not decrease too fast, and so the modulus of the score function can not grow too fast. By considering $S$ normal, so that $\rho$ grows linearly with $u$, we know that the $B^{3}$ rate of growth is a sharp bound.

Lemma 3.4 If $S$ is a random variable with variance $\leq K$, for $X=S+Z_{S}^{(\tau)}$, with score function $\rho$, for $B>1$, there exists a function $f_{1}(\tau, K)$ such that:

$$
\int_{-B \sqrt{\tau}}^{B \sqrt{\tau}} \rho(u)^{2} d u \leq f_{1}(\tau, K) B^{3} .
$$

Proof Now: $p(u) \geq(2 \exp (2 K / \tau))^{-1} \phi_{\tau / 2}(u)$, so that for $u \in(-B \sqrt{\tau}, B \sqrt{\tau})$, $(B \sqrt{\tau} p(u))^{-1} \leq 2 \sqrt{\pi} \exp \left(B^{2}+4 / \tau\right) / B \leq 2 \sqrt{\pi} \exp \left(B^{2}+4 / \tau\right)$. Hence for any $k \geq 1$, by Hölder's inequality:

$$
\begin{aligned}
\int_{-B \sqrt{\tau}}^{B \sqrt{\tau}} \rho(u)^{2} d u & \leq\left(\int_{-B \sqrt{\tau}}^{B \sqrt{\tau}}|\rho(u)|^{2 k} d u\right)^{1 / k}(2 B \sqrt{\tau})^{1-1 / k} \\
& \leq\left(\int_{-B \sqrt{\tau}}^{B \sqrt{\tau}} \frac{p(u)|\rho(u)|^{2 k}}{2 B \sqrt{\tau} \inf _{u} p(u)} d u\right)^{1 / k}(2 B \sqrt{\tau}) \\
& \leq\left(\frac{8 B}{\sqrt{\tau} e}\right) k\left(2 \sqrt{2 \pi} \exp \left(B^{2}+2 K / \tau\right)\right)^{1 / k}
\end{aligned}
$$

Since we have a free choice of $k \geq 1$ to maximise $k \exp (v / k)$, since here $v \geq 1$, taking $k=v$ means that $k \exp (v / k) \exp (-1)=v$. Hence we obtain a bound of

$$
\int_{-B \sqrt{\tau}}^{B \sqrt{\tau}} \rho(u)^{2} d u \leq \frac{8 B}{\sqrt{\tau} e}\left(B^{2}+\frac{2 K}{\tau}+\log (2 \sqrt{2 \pi})\right) \leq \frac{8 B^{3}}{\sqrt{\tau} e}\left(3+\frac{2 K}{\tau}\right) .
$$

We continue by considering $L_{B}=\{|x| \leq B \sqrt{\tau},|y| \leq B \sqrt{\tau}\}$.

Lemma 3.5 For random variables $S, T$, let $X=S+Z_{S}^{(\tau)}$ and $Y=T+Z_{T}^{(\tau)}$. If $S, T$ satisfy the FKG inequalities then there exists a function $f_{2}(\tau, K)$ such that for $B \geq 1$ :

$$
\mathbb{E} M_{a, b}(X, Y) \widetilde{\rho}(X+Y) I\left((X, Y) \in L_{B}\right) \leq f_{2}(\tau, K)(a+b) B^{4} \operatorname{Cov}(S, T) .
$$


Proof Lemma 3 of Newman [13] uses the fact that FKG inequalities imply 'positive quadrant dependence', originally due to Lehman [10]. That is, defining $H(s, t)=\mathbb{P}(S \geq s, T \geq t)-\mathbb{P}(S \geq s) \mathbb{P}(T \geq t), S, T$ are positive quadrant dependent iff $H(s, t) \geq 0$ for all $s, t$, which is a consequence of $S, T$ being FKG. Since $\operatorname{Cov}(S, T)=\int H(s, t) d s d t \geq 0$, then

$$
\operatorname{Cov}(f(S), g(T))=\int f^{\prime}(s) g^{\prime}(t) H(s, t) d s d t \leq\left\|f^{\prime}\right\|_{\infty}\left\|g^{\prime}\right\|_{\infty} \operatorname{Cov}(S, T) .
$$

Since $\left|\phi_{c}(u)^{\prime}\right| \leq \exp (-1 / 2) / \sqrt{2 \pi c^{2}}$, and $\left|\left(u \phi_{c}(u) / c\right)^{\prime}\right| \leq(2 \exp (-3 / 2)) / \sqrt{2 \pi c^{3}}$, we deduce that:

$$
\begin{aligned}
\left|p_{X, Y}(x, y)-p_{X}(x) p_{Y}(y)\right| & \leq \frac{\operatorname{Cov}(S, T)}{2 \pi \tau^{2} e} \\
\left|p_{X, Y}^{(1)}(x, y)-p_{X}^{\prime}(x) p_{Y}(y)\right| & \leq \frac{\operatorname{Cov}(S, T)}{\pi \tau^{5 / 2} e^{2}} \\
\left|p_{X, Y}^{(2)}(x, y)-p_{X}(x) p_{Y}^{\prime}(y)\right| & \leq \frac{\operatorname{Cov}(S, T)}{\pi \tau^{5 / 2} e^{2}} .
\end{aligned}
$$

We can rearrange $M_{a, b}$ to give

$$
\begin{aligned}
M_{a, b}(x, y)= & a\left(\frac{p_{X, Y}^{(1)}(x, y)-p_{X}^{\prime}(x) p_{Y}(y)}{p_{X, Y}(x, y)}\right)+b\left(\frac{p_{X, Y}^{(2)}(x, y)-p_{X}(x) p_{Y}^{\prime}(y)}{p_{X, Y}(x, y)}\right) \\
& +\left(a \rho_{X}(x)+b \rho_{Y}(y)\right)\left(\frac{p_{X}(x) p_{Y}(y)-p_{X, Y}(x, y)}{p_{X, Y}(x, y)}\right) .
\end{aligned}
$$

and hence writing $c$ for $\operatorname{Cov}(S, T) /\left(2 \pi \tau^{5 / 2} e^{2}\right)$, f or $(x, y) \in L_{B}$ :

$$
p_{X, Y}(x, y)\left|M_{a, b}(x, y)\right| \leq c\left(\sqrt{\tau} e\left(a \rho_{X}(x)+b \rho_{Y}(y)\right)+2(a+b)\right) .
$$

By Cauchy-Schwarz:

$$
\begin{aligned}
& \int p_{X, Y}(x, y) M_{a, b}(x, y) \widetilde{\rho}(x+y) I\left((x, y) \in L_{B}\right) d x d y \\
& \leq c \int\left(\sqrt{\tau} e\left(a \rho_{X}(x)+b \rho_{Y}(y)\right)+2(a+b)\right) \widetilde{\rho}(x+y) I\left((x, y) \in L_{B}\right) d x d y \\
& \leq c(a+b)\left(\sqrt{2 B^{4} \sqrt{\tau} f_{1}} \sqrt{16 B^{4} \sqrt{\tau} f_{1}}+\sqrt{4 B^{2} \tau} \sqrt{16 B^{4} \sqrt{\tau} f_{1}}\right)
\end{aligned}
$$


This follows firstly since:

$$
\int \rho_{X}(x)^{2} I\left((x, y) \in L_{B}\right) d x d y \leq(2 B \sqrt{\tau}) \int_{-B \sqrt{\tau}}^{B \sqrt{\tau}} \rho_{X}(x)^{2} d x \leq(2 B \sqrt{\tau}) B^{3} f_{1}(\tau, K) .
$$

and

$$
\begin{aligned}
& \int \widetilde{\rho}(x+y)^{2} I\left((x, y) \in L_{B}\right) d x d y \\
& \leq \int \widetilde{\rho}(x+y)^{2} I(|x+y| \leq 2 B \sqrt{\tau}) I(|y| \leq B \sqrt{\tau}) d x d y \\
& \leq 2 B \sqrt{\tau} \int_{-2 B \sqrt{\tau}}^{2 B \sqrt{\tau}} \widetilde{\rho}(z)^{2} d z \leq 16 B^{4} \sqrt{\tau} f_{1}(\tau, K)
\end{aligned}
$$

Lemma 3.6 For any random variables $S, T$ with mean zero and variance $\leq K$, let $X=S+Z_{S}^{(\tau)}$ and $Y=T+Z_{T}^{(\tau)}$. There exists a function $f_{3}(\tau, K, \epsilon)$ such that:

$$
\mathbb{E} M_{a, b}(X, Y) \widetilde{\rho}(X+Y)\left(I\left((X, Y) \notin L_{B}\right) d x d y \leq(a+b) \frac{f_{3}(\tau, K, \epsilon)}{B^{2-\epsilon}} .\right.
$$

for $S, T$ with $k$ th moment $(k \geq 2)$ bounded above, we can achieve a rate of decay of $1 / B^{k-\epsilon}$.

Proof By Chebyshev $\left.\mathbb{P}\left(\left(S+Z_{S}^{(2 \tau)}, T+Z_{T}^{(2 \tau)}\right) \notin L_{B}\right)\right) \leq \int p^{(2 \tau)}(x, y)\left(x^{2}+\right.$ $\left.y^{2}\right) /\left(2 B^{2} \tau\right) d x d y \leq(K+2 \tau) /\left(B^{2} \tau\right)$ so by Hölder-Minkowski for $1 / p+1 / q=1$ :

$$
\begin{aligned}
& \mathbb{E} \rho_{X, Y}^{(1)}(X, Y) \widetilde{\rho}(X+Y) I\left((X, Y) \notin L_{B}\right) \\
& \quad \leq\left(\mathbb{E}\left|\rho_{X, Y}^{(1)}(X, Y)\right|^{p} I\left((X, Y) \notin L_{B}\right)\right)^{1 / p}\left(\mathbb{E}|\widetilde{\rho}(X+Y)|^{q}\right)^{1 / q} \\
& \left.\quad \leq c_{\tau, p}^{1 / p} c_{\tau, q}^{1 / q} \mathbb{P}\left(\left(S+Z_{S}^{(2 \tau)}, T+Z_{T}^{(2 \tau)}\right) \notin L_{B}\right)\right)^{1 / p} \\
& \quad \leq \frac{2 \sqrt{2} \exp (-1)}{\tau}(2+K / \tau) \sqrt{p q} \frac{1}{B^{2 / p}}
\end{aligned}
$$

By choosing $p$ arbitrarily close to 1 , we can obtain a constant term, as required. The other terms work in a similar way.

Similarly we bound the remaining product term: 
Lemma 3.7 For FKG random variables $S, T$ with mean zero and variance $\leq K$, let $X=S+Z_{S}^{(\tau)}$ and $Y=T+Z_{T}^{(\tau)}$. There exist functions $f_{4}(\tau, K)$ and $f_{5}(\tau, K)$ such that

$$
\mathbb{E} \rho_{X}(X) \rho_{Y}(Y) \leq f_{4}(\tau, K) B^{4} \operatorname{Cov}(S, T)+f_{5}(\tau, K) / B^{2} .
$$

Proof Using part of Lemma 3.5, we know that $p_{X, Y}(x, y)-p_{X}(x) p_{Y}(y) \leq$ $\operatorname{Cov}(S, T) /\left(2 \pi e \tau^{2}\right)$. Hence by argument similar to those of Lemmas 3.6 and 3.7, we obtain that:

$$
\begin{aligned}
\mathbb{E} \rho_{X}(X) \rho_{Y}(Y)= & \int\left(p_{X, Y}(x, y)-p_{X}(x) p_{Y}(y)\right) \rho_{X}(x) \rho_{Y}(y) d x d y \\
\leq & \frac{\operatorname{Cov}(S, T)}{2 \pi e \tau^{2}} \int\left|\rho_{X}(x)\right|\left|\rho_{Y}(y)\right| I\left((x, y) \in L_{B}\right) d x d y \\
& +\int p(x, y)\left|\rho_{X}(x)\right|\left|\rho_{Y}(y)\right| I\left((x, y) \notin L_{B}\right) d x d y \\
& +\int p(x) p(y)\left|\rho_{X}(x)\right|\left|\rho_{Y}(y)\right| I\left((x, y) \notin L_{B}\right) d x d y \\
\leq & \frac{\operatorname{Cov}(S, T)}{2 \pi e \tau^{2}}\left(\int_{-B \sqrt{\tau}}^{B \sqrt{\tau}}\left|\rho_{X}(x)\right|^{2} d x\right)^{2} \\
& +2\left(\int p_{X, Y}(x, y)\left|\rho_{X}(x)\right|^{2} I\left((x, y) \notin L_{B}\right) d x d y\right) .
\end{aligned}
$$

as required.

Proof of Theorem 2.2 Combining Lemmas 3.5, 3.6 and 3.7 , we obtain for given $K, \tau, \epsilon$ that there exist constants $C_{1}, C_{2}$ such that

$$
\mathbb{E} M_{\sqrt{\beta}, \sqrt{1-\beta}} \widetilde{\rho}+\sqrt{\beta(1-\beta)} \mathbb{E} \rho_{X} \rho_{Y} \leq C_{1} \operatorname{Cov}(S, T) B^{4}+C_{2} / B^{2-\epsilon},
$$

so choosing $B=(K / \operatorname{Cov}(S, T))^{1 / 6}>1$, we obtain a bound of $C \operatorname{Cov}(S, T)^{1 / 3-\epsilon}$. By Lemma 3.6, note that if $X, Y$ have bounded $k$ th moment, then we obtain decay at the rate $C_{1} \operatorname{Cov}(S, T) B^{4}+C_{2} / B^{k^{\prime}}$, for any $k^{\prime}<k$. Choosing $B=$ $\operatorname{Cov}(S, T)^{-1 /\left(k^{\prime}+4\right)}$, we obtain a rate of $\operatorname{Cov}(S, T)^{k^{\prime} /\left(k^{\prime}+4\right)}$. Hence for $k \rightarrow \infty$ we can find a rate arbitarily close to $\operatorname{Cov}(S, T)$. 


\section{References}

[1] Barron, A.R. Entropy and the Central Limit Theorem. Annals of Probability, 14:336-342, 1986.

[2] Brown, L.D. A proof of the Central Limit Theorem motivated by the Cramér-Rao inequality. In G. Kallianpur, P.R. Krishnaiah, and J.K. Ghosh, editors, Statistics and Probability: Essays in Honour of C.R. Rao, pages 141-148. North-Holland, New York, 1982.

[3] Carlen, E.A. and Soffer, A. Entropy production by block variable summation and Central Limit Theorems. Communications in Mathematical Physics, 140:339-371, 1991.

[4] Gnedenko, B.V. and Korolev, V.Y. Random Summation: Limit Theorems and Applications. CRC Press, Boca Raton, Florida, 1996.

[5] Grimmett, G.R. Percolation (Second Edition). Springer-Verlag, Berlin, 1999.

[6] Johnson, O.T. Entropy inequalities and the Central Limit Theorem. Stochastic Processes and Their Applications, 88:291-304, 2000.

[7] Johnson, O.T. and Barron, A.R. Information inequalities and the Central Limit Theorem. In preparation, 2001.

[8] Johnson, O.T. and Suhov, Y.M. Entropy and random vectors. Journal of Statistical Physics, 104:147-167, 2001.

[9] Lebowitz, J. GHS and other inequalities. Communications in Mathematical Physics, 35:87-92, 1974.

[10] Lehman, E.L. Some concepts of dependence. Annals of Mathematical Statistics, 37:1137-1153, 1966.

[11] Linnik, Y.V. An information-theoretic proof of the Central Limit Theorem with the Lindeberg Condition. Theory of Probability and Its Applications, 4:288-299, 1959.

[12] Newman, C.M. Moment inequalities for ferromagnetic Gibbs distributions. Journal of Mathematical Physics, 16:1956-1959, 1975. 
[13] Newman, C.M. Normal fluctuations and the FKG inequalities. Communications in Mathematical Physics, 74:129-140, 1980.

[14] Shimizu, R. On Fisher's amount of information for location family. In G.P.Patil et al, editor, Statistical Distributions in Scientific Work, Volume 3, pages 305-312. Reidel, 1975.

[15] Takano, S. The inequalities of Fisher Information and Entropy Power for dependent variables. In S. Watanabe, M. Fukushima, Yu.V Prohorov, and A.N. Shiryaev, editors, Proceedings of the 7th Japan-Russia Symposium on Probability Theory and Mathematical Statistics, Tokyo 26-30 July 1995, pages 460-470, Singapore, 1996. World Scientific.

[16] Takano, S. Entropy and a limit theorem for some dependent variables. In Prague Stochastics '98, volume 2, pages 549-552. Union of Czech Mathematicians and Physicists, 1998. 